\title{
HOLOMORPHIC HULLS AND HOLOMORPHIC CONVEXITY OF DIFFERENTIABLE SUBMANIFOLDS
}

\author{
BY \\ R. O. WELLS, JR. $\left.{ }^{1}\right)$
}

Introduction. The concept of holomorphic hull (or envelope of holomorphy) of a set $K$ on a complex manifold $X$ has been studied extensively in various cases. If $K$ is a domain in $C^{n}$, then the holomorphic hull of $K$ is a well-defined Stein manifold spread over $\boldsymbol{C}^{n}$. There are also many examples of lower dimensional sets $K$ in $C^{n}, n>1$, in which all holomorphic functions on $K$ can be continued analytically to some larger set $K^{\prime} \supset K$. The best known type of example, the boundary of the unit ball, is due to Hartogs, but there are also many examples which are local in nature and indicate that the phenomenon is geometric in nature and not necessarily topological [6], [9], [10].

One basic question is: what is the largest set $K^{\prime}$ to which $K$ can be extended in the above sense of simultaneous analytic continuation? This set, if it exists, should be the holomorphic hull of $K$. In $\S 1$ we define the holomorphic hull of a compact set $K$ in a Stein manifold $X$ to be the spectrum of the algebra of holomorphic functions on $K$ (completed in the maximum norm), which we denote by $E(K)$. $E(K)$ is mapped into $X$ in a natural way, and we see that it is the maximal set to which $K$ can be extended. Moreover, we show that if $E(K)$ is schlicht over $X$, then $K$ can be extended to $E(K)$, thus justifying the definition. If $K=E(K)$, then we say that $K$ is holomorphically convex. In analogy to the theory of polynomially convex sets, there are many problems associated with this concept.

One can give conditions using the Levi form on local submanifolds $M \subset C^{n}, n>1$, that $M$ be locally extendible [1], [7], [9], [16], [18] or that $M$ be locally holomorphically convex [18], [19]. For local hypersurfaces $S$ in $C^{n}$, there are essentially two complementary known results: (1) if the Levi form is nonvanishing at $p \in S$, then the local holomorphic hull of $S$ at $p$ contains an open set, (2) a necessary and sufficient condition that $S$ be locally holomorphically convex is that the Levi form vanishes identically. The problems for lower dimensional manifolds stem from trying to find (1) the structure of local nontrivial hulls (such as being an open set or a higher dimensional manifold), (2) necessary and sufficient geometric conditions that a manifold be locally holomorphically convex, and (3) global conditions that a compact submanifold be extendible or holomorphically convex.

In $\$ 2$ we prove that any submanifold $M$ in $C^{n}$ with a vanishing Levi form is locally holomorphically convex. For a certain class of dimensions the converse is

Received by the editors November 22, 1966 and, in revised form, March 7, 1967.

(1) This research was supported by NSF Grant No. GP-5951. 
true, solving the second problem stated above for this case. In $\$ 3$ we examine in more detail the structure of the local holomorphic hull of an $(n+1)$-dimensional submanifold $M$ in $C^{n}$ with a nonvanishing Levi form. We show that $M$ has a local holomorphic hull which contains an $(n+2)$-dimensional manifold immersed in $\boldsymbol{C}^{n}$ which has the local structure of an $n$-parameter family of Riemann surfaces. We then give an example to show that the local holomorphic hull of such a submanifold need not contain an open set. Bishop [1] shows that, in $C^{3}$, under more stringent conditions, $M$ has a local holomorphic hull which contains an open set. This work has been extended by Weinstock to submanifolds of codimension 2 [17].

In a remark at the end of $\S 3$ we discuss the extendibility of compact submanifolds with constant dimensional complex tangent bundles of positive dimension. It is conjectured that such manifolds are always extendible to higher dimensional manifolds, and some special cases are discussed. On the other hand, one can prove that a compact $k$-dimensional submanifold $M$ with no complex tangent vectors is holomorphically convex (see [20]). In this case one can prove a stronger result, namely that the algebra of holomorphic functions on $M$ is dense in the algebra of continuous functions (see [4], [20], [11]).

1. The holomorphic hull of a compact set. Let $A$ be a uniform algebra of continuous functions on a compact space $K$, and denote by $S(A)$ the spectrum of $A$ (see [5, p. 56]; we shall use [5] as a basic reference throughout this paper).

Let $X$ be a complex manifold with structure sheaf $\mathcal{O}_{X}=\mathcal{O}$ (sheaf of germs of holomorphic functions on $X$ ). If $S$ is a subset of $X$, let $\mathcal{O}(S)$ be the algebra of sections of $\mathcal{O}$ over $S$ (germs of holomorphic functions defined near $S$ ). Let $U$ be an open subset of $X$. Then $\mathcal{O}(U)$ is a Fréchet algebra with the topology of uniform convergence on compact subsets. Let $E(U)$ denote the space of continuous complex homomorphisms of $\mathcal{O}(U)$ into $C$ equipped with the weak-star topology as a subset of the dual of $\mathcal{O}(U)$ (this is the envelope of holomorphy of $U$ if $U$ is contained in a Stein manifold $X$, see [13]).

Let $K$ be a compact subset of $X$, and let $C(K)$ be the Banach algebra of continuous complex-valued functions on $K$ with respect to the maximum norm. Let $\mathscr{H}(K)$ be the subalgebra of $C(K)$ obtained by restricting representatives of elements of $\mathcal{O}(K)$ to $K$ (note that this restriction is not one-to-one), and let $A(K)$ be the closure of $\mathscr{H}(K)$ in $C(K)$.

$A(K)$ is then a uniform algebra on $K$, and we will define the holomorphic hull of $K$ to be $S[A(K)]$, to be denoted by $E(K)$. The justification for this definition will be given by the results of this section.

Proposition 1.1. Suppose $K$ is a compact subset of a Stein manifold $X$, then there are continuous maps

$$
\phi: E(K) \rightarrow X, \quad \pi: K \rightarrow E(K),
$$

where $K \subset \phi[E(K)]$ and $\phi \circ \pi$ is the identity on $K$. 
Proof. Let $\pi: K \rightarrow E(K)$ be the Gel'fand map, where $\pi(x)=\delta_{x}$, the Dirac measure at $x$. Define the map $\phi: E(K) \rightarrow E(X)$ by $\phi(\lambda)=\lambda \mid \mathcal{O}(X)$. It is clear that these are continuous maps and that $\phi \circ \pi$ is the identity on $K$. Applying the theorem that $X$ Stein implies $E(X)=X$ (see [5, p. 222]), we obtain the desired result, and the proposition is proven.

A compact set $K$ in a complex manifold $X$ is a holomorphic set if there is a sequence of open Stein manifolds $X_{i} \subset X$ such that $X_{i+1} \subset X_{i}$ and

$$
K=\bigcap_{i=1}^{\infty} X_{i}
$$

(such sets were called $S_{\delta}$ sets by Rossi [12]). A set $K$ is locally holomorphic at $p \in K$ if there exists a compact neighborhood $N$ of $p$ such that $N \cap K$ is holomorphic. We will say that a set $K$ in a Stein manifold $X$ is holomorphically convex if $K$ is equal to its own holomorphic hull, i.e. if the mapping $\phi$ given by Proposition 1 is a homeomorphism from $E(K)$ onto $K$. And, similarly, a set $K$ is locally holomorphically convex at $p \in K$ if there is a fundamental system of neighborhoods of $p$, whose intersections with $K$ are holomorphically convex. Note that since the intersection of two open Stein submanifolds of $X$ is again an open Stein submanifold, if $K$ is locally holomorphic at $p$, then there is a fundamental sequence of neighborhoods of $p$, whose intersection with $K$ is holomorphic (is a holomorphic set). The following proposition shows that holomorphicity implies holomorphic convexity, and it is unknown to the author whether the converse is true, although it seems likely that that should be the case.

THEOREM 1.2 (ROSSI [12]). If $K$ is a holomorphic set, then $K$ is holomorphically convex.

COROLlaRY 1.3. If $K$ is locally holomorphic at $p \in K$, then $K$ is locally holomorphically convex at $p \in K$.

Suppose $K \subset K^{\prime}$, then we shall say that $K$ is extendible to $K^{\prime}$ if the natural map

$$
r: \mathcal{O}\left(K^{\prime}\right) \rightarrow \mathcal{O}(K)
$$

is an isomorphism. $K$ is said to be extendible, if there exists a $K^{\prime}$ strictly containing $K$, such that $K$ is extendible to $K^{\prime}$.

Proposition 1.4. Suppose $X$ is a Stein manifold. If $K$ is extendible to $K^{\prime}$, then $\phi[E(K)] \supset K^{\prime}$ and the map $\pi: K \rightarrow E(K)$ extends to a map $\pi^{\prime}: K^{\prime} \rightarrow E(K)$ such that $\phi \circ \pi^{\prime}$ is the identity on $K^{\prime}$.

Proof. By hypothesis, $r: \mathcal{O}\left(K^{\prime}\right) \rightarrow \mathcal{O}(K)$ is an algebraic isomorphism. It follows readily that

$$
\|r f\|_{K}=\|f\|_{K^{\prime}}, \quad f \in \mathscr{H}\left(K^{\prime}\right)
$$

and hence that $r$ extends to a topological isomorphism, $r: A\left(K^{\prime}\right) \rightarrow A(K)$. Thus 
$r^{*}: E(K) \rightarrow E\left(K^{\prime}\right)$ is a homeomorphism. Letting $\tilde{\pi}$ be the Gel'fand map mapping $K^{\prime}$ into $E\left(K^{\prime}\right)$, we set $\pi^{\prime}=\left(r^{*}\right)^{-1} \circ \tilde{\pi}$, which proves the proposition.

Assuming that $X$ is still a Stein manifold as before, we make the following definition. We shall say that $E(K)$ is a schlicht holomorphic hull if the map $\phi$ given by Proposition 1.1 is injective, and we set $\phi[E(K)]=\hat{K}$, identifying $E(K)$ and $\hat{K}$ by the homeomorphism $\phi$.

THEOREM 1.3. Suppose $X$ is a Stein manifold and $K$ is a compact subset of $X$ with a schlicht holomorphic hull $\hat{K}$. Then $K$ is extendible to $\hat{K}$.

Proof. Let $U$ be an open set in $X$ containing $K$. Then since $K \subset U \subset X$, the natural restriction maps induce maps

$$
E(K) \stackrel{\tau}{\longrightarrow} E(U) \stackrel{F}{\longrightarrow} E(X)
$$

where $E(U)$ is the envelope of holomorphy of $U$ and $F$ is a locally biholomorphic map (see Rossi [13]). Moreover we have that $F \circ \tau=\phi$. By hypothesis, $\phi$ is injective, hence we obtain that $F \mid \tau(E(K))$ is injective. But $\tau[E(K)]$ is compact, since $E(K)$ is compact. It then follows from the fact that $F$ is locally biholomorphic that there is a neighborhood $N^{\prime}$ of $\tau[E(K)]$ such that $F$ maps $N^{\prime}$ biholomorphically onto a neighborhood $N$ of $\hat{K}$ in $X$, where we have identified $X$ with $E(X)$ since $X$ is Stein. If $f \in \mathcal{O}(U)$, then $f$ extends to $\hat{f} \in \mathcal{O}[E(U)]$, since $E(U)$ is the envelope of holomorphy of $U$. But then $\tilde{f}=\hat{f} \circ F^{-1}$ is holomorphic on $N$, and $\tilde{f}|N \cap U=f| N \cap U$, and $\tilde{f}$ is an extension of $f$ from $U$ to $U \cup N \supset \hat{K}$.

To prove the theorem it suffices to show that the restriction map $r: \mathcal{O}(\hat{K}) \rightarrow \mathcal{O}(K)$ is onto. Suppose $\alpha \in \mathcal{O}(K)$, then there is an open set $U \subset K$ and an $f \in \mathcal{O}(U)$ such that $f$ is a representative for $\alpha$. By the above construction, $f$ can be extended to $\tilde{f} \in \mathcal{O}(N)$ where $N \supset \hat{K}$, and $\tilde{f}$ induces a germ $\beta \in \mathcal{O}(\hat{K})$ such that $r \beta=\alpha$, and the theorem is proven.

2. Local holomorphic convexity of submanifolds in $\boldsymbol{C}^{n}$. Let $M$ be a real submanifold of a complex manifold $X$ (all manifolds will always be $C^{\infty}$ ). We shall say that $M$ is locally extendible at $p \in M$ if, for any sufficiently small neighborhood $N$ of $p, N \cap M$ is extendible. Note that if $M$ is locally extendible at $p \in M$, then $M$ is neither locally holomorphic nor locally holomorphically convex at $p$.

Let $T(M)$ be the tangent bundle to $M$, with fibre $T_{p}(M)$ for $p \in M . T(M)$ is a subbundle of $T(X), X$ considered as a real $C^{\infty}$ manifold, and we will let $J: T(X)$ $\rightarrow T(X)$ be the almost complex tensor given by the complex structure on $X$. Set

$$
T C(M)=T(M) \cap J T(M), \quad T C_{p}(M)=T_{p}(M) \cap J T_{p}(M) .
$$

$T C_{p}(M)$ is a complex subspace of $T_{p}(X)$, and we will call $T C(M)$ the complex tangent bundle to $M$ (it is not always a bundle). The complex rank of TC $(M)$ at $p$ is the complex dimension of $T C_{p}(M)$. We will say that $T C(M)$ is nondegenerate at $p \in M$ if there is a neighborhood $N$ of $p$ such that $T C(N \cap M)$ has constant complex 
rank at each point of $N \cap M . T C(M)$ is nondegenerate on $M$ if it is nondegenerate at each point of $M$. Nondegenerate complex tangent bundles are subbundles of $T(M)$.

At a point where $T C(M)$ is nondegenerate, the Levi form is well defined. Consider the complexification $T C_{p}(M) \otimes C$ (tensor over $R$ ); then $J$ extends naturally to this vector space and has two eigenspaces. Let $T_{p}^{(1,0)}(M)$ be the eigenspace with eigenvalue +1 and let $T_{p}^{(0,1)}(M)$ be the eigenspace with eigenvalue -1 ; then we have $T C_{p}(M) \otimes C=T_{p}^{(1,0)}(M) \oplus T_{p}^{(0,1)}(M)$. Conjugation is well defined here and gives an anti-isomorphism of $T_{p}^{(1,0)}(M)$ onto $T_{p}^{(0,1)}(M)$. Also, there is a canonical isomorphism between $T C_{p}(M)$ and $T_{p}^{(1,0)}(M)$, and we will identify these two vector spaces, using $T C_{p}(M)$ in the sequel.

Definition 2.1. The Levi form is a quadratic form

defined by

$$
L(M): T C(M) \rightarrow C
$$

$$
L_{p}(u)=\pi_{p}[Y, \bar{Y}]_{p}
$$

where $Y$ is a local cross section of $T C(M)$ defined near $p$ such that $Y_{p}=u$ and $\pi_{p}$ is the projection

$$
\pi_{p}: T_{p}(M) \otimes C \rightarrow T_{p}(M) \otimes C / T C_{p}(M) .
$$

Remark. This definition is independent of the choice of $Y$ (cf. [8]).

We shall need the following two results concerning the Levi form on a submanifold of $\boldsymbol{C}^{n}$.

THeOREM 2.2. Let $M$ be a real $k$-dimensional submanifold embedded in $C^{n}$ with a nondegenerate complex tangent bundle of complex rank $m$ at $p \in M$. If the Levi form vanishes identically near $p$, then $M$ can be represented near $p$ as a $(k-2 m)$-parameter family of m-complex-dimensional complex submanifolds of $C^{n}$, i.e. there exists a neighborhood $N$ of $p$, an open set $B$ in $R^{k-2 m} \times C^{m}$, and a map $F: B \rightarrow N$, such that $F$ is an embedding, and if we set

$$
F_{t}(w)=F(t, w), \quad(t, w) \in B, \quad t \in R^{k-2 m}, \quad w \in C^{m},
$$

then $F_{t}$ has maximal complex rank and is holomorphic in

$$
B_{t}=B \cap\left[t \times C^{n}\right]
$$

Proof. Since $T C(M)$ is nondegenerate at $p$ we can find a local basis for $T C(M)$ in a neighborhood $N$ of $p$ in $M$, which we denote by $\left\{X_{1}, \ldots, X_{m}\right\}$. These will be linearly independent complex vector fields of type $(1,0)$ on $M$ defined near $p$. The vector fields $\left\{\operatorname{Re} X_{j}, \operatorname{Im} X_{j}, j=1, \ldots, m\right\}$ span $T C(M)$ (locally) when considered as a real subbundle of $T(M)$ and we can extend them to a basis of $T(M)$ by adjoining $l$ real vector fields $\left\{Y_{1}, \ldots, Y_{l}\right\}$ where we have set $l=k-2 m$. Suppose $Y$ is a local cross section of $T C(M)$, then we have

$$
Y=\sum_{j=1}^{m} \alpha_{i} X_{j}
$$


and hence

$$
[Y, \bar{Y}]=\sum_{i, j=1}^{m} \alpha_{i} \bar{\alpha}_{j}\left[X_{i}, \bar{X}_{j}\right]
$$

Moreover, we have that each commutator on the right-hand side is a vector field on $M$, and we obtain

$$
\left[X_{i}, \bar{X}_{j}\right]=\sum_{\mu=1}^{m}\left(b_{i j}^{\mu} X_{\mu}+c_{i j}^{\mu} \bar{X}_{\mu}\right)+\sum_{v=1}^{l} d_{i j}^{v} Y_{v}
$$

(we have expressed $\left\{\operatorname{Re} X_{\mu}, \operatorname{Im} X_{\mu}\right\}$ in terms of $\left\{X_{\mu}, \bar{X}_{\mu}\right\}$ ). Thus the Levi form vanishing identically near $p$ implies that $d_{i j}^{v}$ vanish identically near $p$. Then (2.2) gives the complex integrability conditions of Sommer [15], and Sommer proves in his paper that the integrability conditions are necessary and sufficient that the submanifold $M$ be fibred into an $l$-parameter family of $m$-complex dimensional complex submanifolds of $C^{n}$. The theorem is then proven.

THEOREM $2.3[18]\left({ }^{2}\right)$. Let $M$ be a real $k$-dimensional submanifold embedded in $C^{n}$, with $k>n$ and complex rank $T C(M)=k-n$. If

(a) $T C(M)$ is nondegenerate at $p$, and $L_{p}(u) \neq 0$ for all $u \in T C_{p}(M)$, or

(b) there are no positive dimensional subvarieties of $C^{n}$ embedded in $M$ passing through $p$, then $M$ is locally extendible at $p$.

We now have our main result concerning local holomorphic convexity of submanifolds in $\boldsymbol{C}^{n}$.

THEOREM 2.4. Let $M$ be a real submanifold of $C^{n}$ with a nondegenerate complex tangent bundle at $p \in M$. Suppose the Levi form vanishes identically on $M$ near $p$, then $M$ is locally holomorphic at $p$.

First we have the following simple lemmas.

LeMma 2.5. Suppose $M$ is a real $k$-dimensional submanifold of $C^{n}$ with rank $T C_{p}(M)=m$, for $p \in M$. Let $s=k-2 m, r=n+m-k$. After a complex-linear change of coordinates in $C^{n}, M$ may be represented as a map $F: N \rightarrow C^{n}$, where $N$ is a neighborhood of the origin in $R^{s} \times C^{m}$, and

$$
\begin{aligned}
F^{j}(t, w) & =t_{j}+i g^{j}(t, w), \quad j=1, \ldots, s, \\
F^{j+s}(t, w) & =h^{j}(t, w), \quad j=1, \ldots, r, \\
F^{j+s+r}(t, w) & =w_{j}, \quad j=1, \ldots, m,
\end{aligned}
$$

$(t, w)=\left(t_{1}, \ldots, t_{s}, w_{1}, \ldots, w_{m}\right)$ are coordinates in $R^{s} \times C^{m}$, and $\left\{g^{j}\right\},\left\{h^{j}\right\}$ are real and complex-valued functions respectively, vanishing to second order at $(t, w)=0$.

$\left({ }^{2}\right)$ This theorem was stated more generally in [18], but the proof given was only applicable to the case stated here. 
Proof. This follows easily from elementary linear algebra and the implicit function theorem, see [1], [18].

LEMmA $2.5^{\prime}$. Let $U$ be a domain in $C^{n}$, and let $\phi$ be a real-valued function defined in $U$, and let

$$
S=\{x: \phi(x)=0\}
$$

be a hypersurface in $U$ with $d \phi \neq 0$ on $S$. Let $p$ be a point on $S$ and suppose that there is a complex submanifold $Y$ of $C^{n}$ of complex dimension $m$ embedded in $S$ passing through $p$. Then the complex Hessian of $\phi$,

$$
H=H(\phi)=\left(\frac{\partial^{2} \phi}{\partial z_{i} \partial \bar{z}_{j}}\right)
$$

has at least $m$ zero eigenvalues at $p$ (with eigenvectors in $T C_{p}(S)$ ).

Proof. Let $z=\left(z_{1}, \ldots, z_{n}\right)$ be the given coordinates in $C^{n}$, and assume that $p$ is $z=0$. There is a holomorphic change of variables $z=g(\zeta)$, such that (i) $A=$ (dg evaluated at $z=0$ ) is unitary, (ii) (setting $\left.\zeta_{j}=\xi_{j}+i \eta_{j}\right) T_{p}(S)=\left[\eta_{n}=0\right], T C_{p}(S)=$ $\left[\zeta_{n}=0\right]$, and (iii) in a neighborhood $U^{\prime}$ of $z=0$ we have

$$
Y \cap U^{\prime}=\left[\zeta_{m+1}=\cdots=\zeta_{n}=0\right] .
$$

Thus if we set $\phi(\zeta)=\phi[g(\zeta)]$ and let $\tilde{H}$ be the Hessian of $\tilde{\phi}$ in the $\zeta$ coordinates, we have

$$
\tilde{H}=\bar{A}^{*} H \bar{A}
$$

where * denotes Hermitian adjoint. Since $A$ is unitary we see that $H$ and $\tilde{H}$ have the same eigenvalues. It is easy to see that $H$ has at least $m$ zero eigenvalues with eigenvectors in $T C_{p}(S)$ since

$$
\partial^{2} \tilde{\phi} / \partial \zeta_{i} \partial \bar{\zeta}_{j}=0, \quad i \leqq m, j \leqq n ; i \leqq n, j \leqq m,
$$

which follows from (2.4). The lemma is proven.

Proof of Theorem 2.4. Set $s=k-2 m, r=n+m-k$. By Theorem 2.2 we see that $M$ is (near $p$ ) an $s$-parameter family of $m$-complex dimensional complex submanifolds of $C^{n}$. Let $B_{\rho}$ be a ball of radius $\rho$ about $p$, so that each point $q$ of $B_{\rho} \cap M$ has an $m$-dimensional complex submanifold embedded in $M$ passing through $q$. Assume the representation for $M$ given by Lemma 2.5 and identify

$$
\left(x_{1}, \ldots, x_{s}\right)=\left(t_{1}, \ldots, t_{s}\right), \quad\left(z_{s+r+1}, \ldots, z_{n}\right)=\left(w_{1}, \ldots, w_{m}\right)
$$

where $z_{j}=x_{j}+i y_{j}, j=1, \ldots, n$, are coordinates in $C^{n}$. Set

$$
g^{j}(z)=g^{j}(t, w), \quad h^{j}(z)=h^{j}(t, w)
$$

and assume that $\rho$ is chosen so that $\left\{g^{j}\right\}$ and $\left\{h^{j}\right\}$ are well defined in $B_{\rho}$. 
Consider a local tubular neighborhood $N_{\varepsilon}$ of $M$ of radius $\varepsilon$ given in the following manner: Let $T_{0}(M)^{\perp}$ denote the orthogonal complement to $T_{0}(M)$ in $C^{n}$, and define

$$
N_{\varepsilon}=\left\{z \in C^{n}: z=p+v, p \in M, v \in T_{0}(M)^{\perp},|v|<\varepsilon\right\} .
$$

If we define

$$
\phi(z)=\sum_{j=1}^{s}\left[y_{j}-g^{j}(z)\right]^{2}+\sum_{j=1}^{r}\left|z_{j}-h^{j}(z)\right|^{2},
$$

we then have

$$
B_{\rho} \cap M=\left\{z \in B_{\rho}: \phi(z)=0\right\}, \quad B_{\rho} \cap \partial N_{\varepsilon}=\left\{z \in B_{\rho}: \phi(z)=\varepsilon\right\} .
$$

Let

where

$$
H(\phi)=H=\left[\begin{array}{ccc}
A_{s}(z) & * & * \\
* & B_{r}(z) & * \\
* & * & C_{m}(z)
\end{array}\right],
$$

$$
\begin{aligned}
A_{s} & =\partial^{2} \phi / \partial z_{i} \partial \bar{z}_{j}, & & i, j=1, \ldots, s, \\
B_{r} & =\partial^{2} \phi / \partial z_{i} \partial \bar{z}_{j}, & & i, j=s+1, \ldots, s+r, \\
C_{m} & =\partial^{2} \phi / \partial z_{i} \partial \bar{z}_{j}, & & i, j=s+r+1, \ldots, n .
\end{aligned}
$$

We have the following estimates, letting $I_{l}$ be the $l \times l$ identity matrix:

$$
\begin{gathered}
A_{s}=\frac{1}{2} I_{s}+O(|z|) \\
B_{r}=I_{r}+O(|z|) \\
C_{m}=O(|z|) \\
H(\phi)-\left[\begin{array}{lll}
\frac{1}{2} I_{s} & 0 & 0 \\
0 & I_{r} & 0 \\
0 & 0 & 0
\end{array}\right]=O(|z|) .
\end{gathered}
$$

Hence we obtain the result that at $z=0, H$ has at least $s+r$ positive eigenvalues. It follows that $\rho$ can be chosen so that $H$ has at least $s+r$ positive eigenvalues on $\bar{B}_{2 \rho}$. The sets

$$
D_{\varepsilon}=\left\{z \in B_{\rho+\varepsilon}: \phi(z)<\varepsilon\right\}
$$

are neighborhoods of $M \cap \bar{B}_{\rho}$ with piecewise smooth boundaries such that

$$
\bigcap_{\varepsilon>0} D_{\varepsilon}=M \cap \bar{B}_{\rho} \text {. }
$$

We shall now show that for sufficiently small $\varepsilon$, each $D_{\varepsilon}$ is a pseudoconvex domain. For $\rho$ and $\varepsilon$ small enough $N_{\varepsilon}$ will have a smooth boundary. Suppose this is the case. Also choose $\varepsilon<\rho$. To show that $D_{\varepsilon}$ is pseudoconvex it suffices to show that $D_{\varepsilon}$ is bounded by pseudoconvex surfaces. We have

$$
\partial D_{\varepsilon}=\partial B_{\rho+\varepsilon} \cap[\phi \leqq \varepsilon] \cup \bar{B}_{\rho+\varepsilon} \cap[\phi=\varepsilon]
$$


and it suffices to show that the surface $S=[\phi=\varepsilon]$ is pseudoconvex with respect to the side $[\phi<\varepsilon]$. By construction $d \phi \neq 0$ on $S$.

Points $p$ on $S$ are of the form $p_{0}+v$ where $v \in T_{0}(M)^{\perp},|v|<\varepsilon$ and $p_{0} \in M$. Hence for fixed $v$, we have a neighborhood of $p_{0}$ in $M$ is translated to a submanifold of $S$ passing through $p$. Since we have

$$
\left|p_{0}\right|<|p|+|v|<\rho
$$

there is a complex submanifold of $S$ of complex dimension $m$ passing through $p$. Hence by Lemma $2.5^{\prime}$ we obtain that $H(\phi-\varepsilon)=H(\phi)$ has at least $m$ zero eigenvalues at $p$. But we know also that there are at least $r+s$ positive eigenvalues of $H(\phi)$ at $p$, and since $n=r+s+m$, we see that $H(\phi)$ has only nonnegative eigenvalues in $T C_{p}(S)$, and hence the surface $S$ is pseudoconvex at $p$ with respect to the side $[\phi<\varepsilon]$. Thus $D_{\varepsilon}$ is a pseudoconvex domain and by the solution to the Levi problem it is an open Stein submanifold of $C^{n}$ (see [5, Chapter IX]). Hence $M$ is locally holomorphic, and the theorem is proven.

COROLLARY 2.6. Let $M$ be a real submanifold of $C^{n}$ with a nondegenerate complex tangent bundle at $p \in M$. If the Levi form vanishes identically near $p$, then $M$ is locally holomorphically convex at $p$.

Proof. Apply Corollary 1.3.

THeOREM 2.7. Suppose $M$ is a real $(n+1)$-dimensional submanifold of $C^{n}$ and suppose $M$ has a nondegenerate complex tangent bundle at $p \in M$ of complex rank 1 . Then $M$ is locally holomorphic at $p$ if and only if the Levi form vanishes identically near $p$.

REMARK. This theorem was proved for hypersurface in [18], and for 4-dimensional real-analytic submanifolds of $C^{3}$ in [19].

Proof. Suppose the Levi form does not vanish identically near $p$. Then for any neighborhood $N$ of $p$, there exists a point $q \in N \cap M$ such that $L_{q}(M) \neq 0$. Then Theorem 2.3 implies that $N \cap M$ cannot be holomorphic. Since $N$ was arbitrary, this shows that $M$ is not locally holomorphic at $p$ when the Levi form does not vanish identically near $p$. However, if the Levi form does vanish identically near $p$, then Theorem 2.4 is applicable and the theorem is proven.

COROLLARY 2.8. Under the same assumptions on $M$ as in Theorem 2.7, a necessary and sufficient condition for $M$ to be locally holomorphically convex at $p \in M$ is that the Levi form vanish identically near $p$.

Proof. Sufficiency is given by Corollary 2.6. Necessity follows as in the proof of Theorem 2.7 where we use the fact that an extendible set cannot be holomorphically convex as we see immediately from Proposition 1.4 and the definition of holomorphic convexity. 
REMARK. It is likely that Theorem 2.7 and its corollary are true without the dimensional restrictions presently necessary.

3. Structure of local holomorphic hulls. Let $M$ be a real $C^{\infty}(n+1)$-dimensional submanifold embedded in $C^{n}$, where $n$ is fixed and $\geqq 2$. Suppose $T C(M)$ is nondegenerate at $p \in M$. We shall assume that rank $T C(M)$ at $p$ is one. We have first a $C^{\infty}$ embedding $\phi$

$$
\phi: \bar{B} \subset R^{n-1} \times C^{1} \rightarrow C^{n}
$$

where $B$ is the unit ball in $R^{n-1} \times C^{1}$, and $\phi(B)=M$. Letting $(u, w)$ be the coordinates in $R^{n-1} \times C^{1}$, we obtain, as in Lemma 2.5,

$$
\begin{aligned}
\phi^{j}(u, w) & =u_{j}+i h^{j}(u, w), \quad j=1, \ldots, n-1, \\
\phi^{n}(u, w) & =w,
\end{aligned}
$$

where the real-valued $C^{\infty}$ functions $h^{i}$ vanish to second order at $(u, w)=(0,0)$.

Let $D$ be the unit disc $[|\zeta|<1]$ in the complex $\zeta$ plane, and let $I$ be the unit interval $[0,1]$. Consider a continuous map $f$ where

$$
f: \partial D \times I^{n} \rightarrow B \text {. }
$$

Then $\tilde{f}=\phi \circ f$ is an $n$-parameter family of closed curves on $M$. By a "perturbation" of the map $f$ to a new $\operatorname{map} f_{1}$, we can find a map $F$ where

such that

$$
F: \bar{D} \times I^{n} \rightarrow C^{n}
$$

(i) For fixed $t \in I^{n}, F(\zeta, t)$ is holomorphic at $\zeta \in D$ and has complex rank 1 .

(ii) $F \mid \partial D \times I^{n}=f_{1} \circ \phi$.

This is Bishop's result [1]. We want to extend Bishop's work to show that $F$ can be taken to be a differentiable mapping on $\bar{D} \times I^{n}$ which, under appropriate convexity conditions on $M$, has maximal rank at all points of $\bar{D} \times I^{n}$ except for lower dimensional sets.

Let $T$ be the Hilbert transform of $C^{\infty}$ real-valued functions $u$ on $\partial D$ defined as follows: $u=\operatorname{Re}(G) \mid \partial D$, where $G$ is holomorphic in $D$ and $\operatorname{Im}(G)(0)=0$. Let $T u=\operatorname{Im}(G) \mid \partial D$. Let

$$
\begin{aligned}
I_{0} & =[0,1], I=[-1,1], \\
Q & =\bar{D} \times I_{0} \times I^{n-1}, \\
P & =\partial D \times I_{0} \times I^{n-1},
\end{aligned}
$$

with coordinates in $Q$ given by $q=\left(\rho e^{i \theta}, r, t\right)$ and in $P$ by $p=\left(e^{i \theta}, r, t\right)$, $t=\left(t_{1}, \ldots, t_{n-1}\right)$.

To find a mapping $F: Q \rightarrow C^{n}$ which has the above properties it suffices to solve the following equation

$$
f(p)=\varepsilon t-T h[f(p), g(p)]
$$

for $f$. Here $f$ is a map,

$$
f: P \rightarrow R^{n-1},
$$


$g(p)$ is a given complex-valued function on $P$ such that, for each fixed $(r, t), g(p)$ is the trace on $\partial D$ of a holomorphic function $\tilde{g}$ on $D$, and $\varepsilon>0$ is a fixed arbitrary constant. Suppose $f$ satisfies (3.2), then it follows immediately that for each fixed $(r, t)$

$$
f(p)+i h[f(p), g(p)]
$$

is the trace of a holomorphic map $f$

$$
f: D \rightarrow C^{n-1} \text {. }
$$

Therefore the pair $(f, g)$ is the restriction to $P$ of a map

$$
F: Q \rightarrow C^{n}
$$

which is holomorphic for fixed $(r, t)$. Thus we must solve (3.2) so that the solution $f$ is in $C^{k}$ in order to obtain a differentiable manifold $F(Q)$ (of order $k$ ) at those points in $Q$ where $F$ has maximal rank.

Consider the Sobolev spaces $H^{m}(P)$, which consist of $L^{2}$ functions defined on $P$ with distribution derivatives up to order $m$ also in $L^{2}(P)$. It is well known that

$$
H^{m}(P) \subset C^{k}(\bar{P})
$$

for $2 k<2 m-\operatorname{dim} P$ (see e.g. [3, p. 232]). We consider vector-valued functions

$$
f=\left(f_{1}, \ldots, f_{l}\right) \in\left[H^{m}(P)\right]^{l}=H_{l}^{m}(P) .
$$

Denote the norm in $H^{m}(P)$ by \|\|$_{m}$ and use the same notation for $f \in H_{l}^{m}(P)$, i.e.

$$
\|f\|_{m}=\left\|f_{1}\right\|_{m}+\cdots+\left\|f_{l}\right\|_{m} .
$$

Let \|\|$_{P}$ denote the maximum norm over $P$ and we treat vector-valued functions as above. For $f \in H_{l}^{m}(P), m>(n+1) / 2$,

$$
\|f\|_{P} \leqq C_{m}\|f\|_{m},
$$

where $C_{m}$ depends on $l$. In the applications here, $l$ will always be either $n-1$ or $n+1$ and we shall assume $C_{m}$ is the same for both cases. Assume also that $B=$ $\left\{(u, w):\|(u, w)\|_{P}<1\right\}$.

Lemma 3.1. Let $f, g \in H_{n+1}^{m}(P),\|f\|_{m} \leqq 1,\|g\|_{m} \leqq 1, m>2[(n+1) / 2]\left({ }^{3}\right)$

$$
f: P \rightarrow B, \quad g: P \rightarrow B,
$$

then there exists a constant $K_{m}$ depending only on $B, h$, and $m$ such that

$$
\begin{aligned}
\|h(f)\|_{m} & \leqq K_{m}\|f\|_{m}^{2}, \\
\|h(f)-h(g)\|_{m} & \leqq K_{m}\|f-g\|_{m}\left(\|f\|_{m}+\|g\|_{m}\right) .
\end{aligned}
$$

Proof. These inequalities are derived by Bishop [1] in the case $m=1$ without norms on the parameters. However, the arguments extend easily to this case and we will omit the details here.

(3) [ ] denotes greatest integer. 
Take $K_{m}>C_{m} / 2$ in Lemma 3.1, and let $\gamma=\|t\|_{m}$ which is a constant independent of $m, m \geqq 1$. We now have the basic existence theorem. This theorem is due to Bishop [1] in the case $m=1$, when the $L^{2}$ norms are taken only over the unit circle. The somewhat stronger result presented here gives us differentiability with respect to the parameters involved.

THEOREM 3.2. Suppose $m>2[(n+1) / 2]$ and

$$
0<\varepsilon_{0}<\min \left\{\left(2 C_{m}\right)^{-1},(16 \gamma)^{-1}\right\} .
$$

If $g \in H_{2}^{m}(P), g: P \rightarrow C$, with $\|g\|_{m} \leqq \varepsilon_{0}$, then there exists uniquely an $f \in H_{n-1}^{m}(P)$ such that

(i) $(f, g): P \rightarrow B$,

(ii) $f(p)=\varepsilon_{0} t-T h(f(p), g(p))$.

Proof. Let $f^{0}(p)=\varepsilon_{0}$, and define by induction

$$
f^{j}(p)=\varepsilon_{0} t-T h\left(f^{j-1}(p), g(p)\right) .
$$

Using the inequalities in Lemma 3.1, it is easy to see that $f^{j}$ converges in $H_{n-1}^{m}(P)$ to $f \in H_{n-1}^{m}(P)$ which satisfies the equation in (ii) and satisfying

$$
\|f\|_{m} \leqq\left(4 K_{m}\right)^{-1}
$$

(see [1]). Then we have that

Hence

$$
\begin{aligned}
\|(f, g)\|_{P} & \leqq C_{m}\|(f, g)\|_{m} \\
& =C_{m}\left(\|f\|_{m}+\|g\|_{m}\right) \\
& \leqq C_{m} / 4 K_{m}+\frac{1}{2}<1 .
\end{aligned}
$$

$$
(f, g): B \rightarrow P
$$

and the theorem is proven.

COROLlARY 3.3. If $(\varepsilon, g)$ and $(\hat{\varepsilon}, \hat{g})$ are two sets of data with $m$-norm $<\varepsilon_{0}$, and if $f$, $\hat{f}$ are the corresponding solutions of (3.2), then

$$
\|f-\hat{f}\|_{m} \leqq 4 \gamma\left\{|\varepsilon-\hat{\varepsilon}|+\|g-\hat{g}\|_{m}\right\} .
$$

Proof. We have

$$
f-\hat{f}=\varepsilon t-\hat{\varepsilon} t+T[h(f, g)-h(\hat{f}, \hat{g})]
$$

and hence

$$
\begin{aligned}
\|f-\hat{f}\|_{m} & \leqq \gamma|\varepsilon-\hat{\varepsilon}|+K_{m}\left\{\|f-\hat{f}\|_{m}+\|g-\hat{g}\|_{m}\right\} \cdot\left\{\|f\|_{m}+\|\hat{f}\|_{m}+\|g\|_{m}+\|\hat{g}\|_{m}\right\} \\
& \leqq|\varepsilon-\hat{\varepsilon}| \gamma+\frac{3}{4}\left\{\|f-\hat{f}\|_{m}+\|g-\hat{g}\|_{m}\right\} .
\end{aligned}
$$

Therefore,

$$
\|f-\hat{f}\|_{m} \leqq 4 \gamma\left\{|\varepsilon-\hat{\varepsilon}|+\|g-\hat{g}\|_{m}\right\},
$$

as desired. 
Assume now that $m>2[(n+1) / 2]$, let $\varepsilon_{0}$ be given as in Theorem 3.2, and let $\varepsilon$ be such that $0<\varepsilon \leqq \varepsilon_{0}$. Let

$$
Q_{\varepsilon}=\bar{D} \times I_{0}(\varepsilon) \times I^{n-1}(\varepsilon), \quad P_{\varepsilon}=\partial D \times I_{0}(\varepsilon) \times I^{n-1}(\varepsilon),
$$

where $I_{0}(\varepsilon)=[0, \varepsilon], I(\varepsilon)=[-\varepsilon, \varepsilon]$. Define $g: P \rightarrow C$ by

$$
g(p)=g\left(e^{i \theta}, s\right)=\varepsilon r e^{i \theta} .
$$

Consider the mapping $\psi=\phi \circ(f, g)$ where $f$ is the solution to (3.2) for $g, \varepsilon$ as given here. Let $c=\varepsilon t, s=\varepsilon r$, and we have then

$$
f(p)=c-T h[f(p), s \tau],
$$

where $p=\left(e^{i \theta}, s, c\right)$ are now the coordinates in $P_{\varepsilon}$. Thus $\psi$ has the form

$$
\begin{aligned}
& \psi_{j}(p)=f^{j}(p)+i h^{j}(f(p), g(p)), \quad j=1, \ldots, n-1, \\
& \psi_{n}(p)=g(p),
\end{aligned}
$$

and maps $P_{\varepsilon}$ to $M$. Setting $\tau=e^{i \theta}$, we define

$$
F_{j}(\zeta, c, s)=\frac{1}{2 \pi i} \int_{\partial D} \frac{\psi_{j}(\tau, c, s)}{\tau-c} d \tau,
$$

where $\zeta \in D$. This defines a mapping

$$
F: Q_{\varepsilon} \rightarrow C^{n}
$$

where $F \in C^{k}\left(Q_{\varepsilon}\right)$ since $(f, g) \in C^{k}\left(P_{\varepsilon}\right), k \geqq 1$. We use here the fact that if for fixed ( $c, s), f$ satisfies (3.3), then $\psi_{j}, j=1, \ldots, n-1$, are the boundary values of holomorphic functions which are then given as above by the Cauchy integral formula. Thus we obtain a differentiable map (3.4), where the order of the differentiability depends on the size of $\varepsilon$.

Lemma 3.4. Let $M \subset C^{n}$ be given as in (3.1). Then the Levi form $L_{p} \neq 0$ at $p$ if and only if

for some $j, j=1, \ldots, n-1$.

$$
\left.h_{w \bar{w}}^{j}\right|_{0} \neq 0
$$

Proof. There is a vector field $X$ of type $(1,0)$ which generates $T C(M)$ near $p$. Let

$$
\left(x_{1}, \ldots, x_{n+1}\right)=\left(u_{1}, \ldots, u_{n-1}, \operatorname{Re} w, \operatorname{Im} w\right)
$$

be the real local coordinates on $M$ as given by the embedding $\psi$

$$
\partial \phi^{i} / \partial x_{j}=\phi_{j}^{i}, \quad \partial h^{i} / \partial x_{j}=h_{j}^{i}
$$

$X$ is then given by

$$
X=\sum_{j=1}^{n+1} \psi_{j} \frac{\partial}{\partial x_{j}}
$$

where the $\left\{\psi_{j}\right\}$ must satisfy

$$
\sum_{j=1}^{n+1} \psi_{j} \phi_{j}^{i}=0, \quad i=1,2,3 .
$$


Since the matrix $\left(\phi_{j}^{i}\right), i, j=1, \ldots, n$ is nonsingular near $p$, we may set $\psi_{n+1}=1$ and solve for $\psi_{j}, j=1, \ldots, n$.

Now $L_{p} \neq 0$ at $p$ means that the vector fields $[X, \bar{X}], X, \bar{X}$ are linearly independent at $p$. This implies that the matrix $D$ given by

$$
D=\left[\begin{array}{c}
\psi_{j} \\
\bar{\psi}_{j} \\
X \bar{\psi}_{j}-\bar{X} \psi_{j}
\end{array}\right], \quad j=1, \ldots, n+1,
$$

has rank 3 at $p$ (and hence near $p$ ). By solving (3.5) explicitly for $\psi_{j}$ we obtain at $p$, since $\left.h_{j}^{i}\right|_{p}=0$,

$$
D=\left[\begin{array}{lllrl}
0 & & 0 & i & 1 \\
0 & \cdots & 0 & -i & 1 \\
\alpha_{1} & & \alpha_{n-1} & 0 & 0
\end{array}\right]
$$

where

$$
\alpha_{j}=\left.\left(X \psi_{j}-\bar{X} \psi_{j}\right)\right|_{p}, \quad j=1, \ldots, n-1 .
$$

A further calculation then gives us that

$$
\alpha_{j}=-8 i\left(h_{w \bar{w}}^{j}\right), \quad j=1, \ldots, n-1 .
$$

Thus, it is clear that a necessary and sufficient condition that $L_{P} \neq 0$ at $p$ is that $h_{w \bar{w}}^{j}$ be nonzero at $p$ for some $j, j=1, \ldots, n-1$.

By virtue of Lemma 3.4, we may assume that for $L_{p} \neq 0$ at $p \in M^{n+1}$ the mapping $\phi$ is of the form (where $p=0$ in $C^{n}$ )

$$
\begin{aligned}
& \phi^{1}=u_{1}+i w \bar{w}+O\left(|x|^{3}\right), \\
& \phi^{j}=u_{j}+i O\left(|x|^{3}\right), \quad j=2, \ldots, n-1, \\
& \phi^{3}=w .
\end{aligned}
$$

(See [1, p. 15]; the nonvanishing of the Levi form is then equivalent to Bishop's first assumption.)

We now want to compute the rank of the Jacobian of the map $F$. Set $\zeta=\xi+i \eta$, $F_{j}=U_{j}+i V_{j}$, and

$$
J(F)=\left[\begin{array}{llllll}
\frac{\partial U_{j}}{\partial \xi} & \frac{\partial U_{j}}{\partial \eta} & \frac{\partial U_{j}}{\partial s} & \frac{\partial U_{j}}{\partial c_{1}} & \cdots & \frac{\partial U_{j}}{\partial c_{n-1}} \\
\frac{\partial V_{j}}{\partial \xi} & \frac{\partial V_{j}}{\partial \eta} & \frac{\partial V_{j}}{\partial s} & \frac{\partial V_{j}}{\partial c_{1}} & \cdots & \frac{\partial V_{j}}{\partial c_{n-1}}
\end{array}\right],
$$

$j=1, \ldots, n$. Let $S(F)$ denote the set of singularities of $F$; i.e. the set of points in $Q_{\varepsilon}$ where $J(F)$ has rank less than $n+2$. We shall show that $S$ is a lower dimensional set. 
LEMMA 3.5 .

$$
\begin{array}{rlrl}
\partial U_{j} /\left.\partial c_{k}\right|_{\zeta=0} & =\delta_{j k}, & & j, k=1, \ldots, n-1, \\
\partial U_{j} /\left.\partial s\right|_{\zeta=0} & =0, & & j=1, \ldots, n-1, \\
\partial V_{j} /\left.\partial c_{k}\right|_{\zeta=0} & =O(|p|), & j, k=1, \ldots, n-1 .
\end{array}
$$

Proof. We have, for $j=1, \ldots, n-1$

$$
\begin{aligned}
F_{j}(0, c, s) & =\frac{1}{2 \pi i} \int_{\partial D} \frac{\psi_{j}(\tau, c, s)}{\tau} d \tau \\
& =c_{j}+\frac{i}{2 \pi} \int_{0}^{2 \pi} h^{j}\left(f\left(e^{i \theta}, e, s\right), g\left(e^{i \theta}, s\right)\right) d \theta .
\end{aligned}
$$

Thus the first two parts of the lemma are clear. We now compute

$$
\frac{\partial V_{j}}{\partial c_{k}}=\frac{1}{2 \pi} \int_{0}^{2 \pi} \sum_{i=1}^{n-1} h_{i}^{j}(f, g) \frac{\partial f_{i}}{\partial c_{k}} d \theta .
$$

Let $C$ denote large constants. Then we have

$$
\left\|\frac{\partial f_{i}}{\partial c_{j}}\right\|_{P} \leqq C\|f\|_{m}, \quad\|f\|_{p} \leqq C\|f\|_{m}
$$

We obtain easily the following inequality

$$
\|(f, g)\|_{P} \leqq C|p| .
$$

This follows from Corollary 3.3 and the fact that $\|g\|_{m} \leqq C\|g\|_{P} \leqq C|p|$. Remembering that $h_{i}^{j}(0,0)=0$, we obtain

$$
\left\|h_{i}^{j}(f, g)\right\|_{P} \leqq C\|(f, g)\|_{P},
$$

which, with (3.7), give the desired result.

LEMMA 3.6.

$$
\partial V_{1} /\left.\partial s\right|_{\zeta=0}=s+O\left(|p|^{2}\right)
$$

Proof. Let $w=w_{1}+i w_{2}$. Then we have from (3.6) that

$$
h_{i}^{l}=2 w_{i+1-n}\left(\delta_{i, n}+\delta_{i, n+1}\right) O\left(|x|^{2}\right), \quad i=1, \ldots, n+1 .
$$

Differentiating the equation

$$
V_{1}(0, c, s)=(1 / 2 \pi) \int h^{1}(f, g) d \theta
$$

with respect to $s$ and using (3.8) we obtain easily the estimate (setting $g=g_{1}+i g_{2}$ )

$$
\left.\frac{\partial V_{1}}{\partial s}\right|_{\tau=0}=s+\frac{1}{2 \pi} \int\left[\sum_{i=1}^{n-1} \frac{\partial f_{i}}{\partial s} O\left(|x|^{2}\right)+\sum_{i=1}^{2} \frac{\partial g_{i}}{\partial s} O\left(|x|^{2}\right)\right] d \theta
$$

Since $\partial f_{i} / \partial s$ and $\partial g_{i} / \partial s$ are bounded, we obtain the desired result, using (3.7), and the fact that $x$ is evaluated at $(f, g)(p)$. 
Lemma 3.7. There exists an $\varepsilon_{1}>0$ such that, for $|p|<\varepsilon_{1}$,

$$
\left.\operatorname{Rank} J(F)\right|_{\zeta=0}=n+2 .
$$

Proof. Since $g(s, \zeta)=s(\xi+i \eta)$, we have the last two rows of $J(F)_{\zeta=0}$ obtained from $U_{n}$ and $V_{n}$ given by

$$
\left[\begin{array}{lllll}
s & 0 & 0 & \cdots & 0 \\
0 & s & 0 & \cdots & 0
\end{array}\right]
$$

Hence it suffices to show that the matrix

$$
M=\left[\begin{array}{ll}
\frac{\partial U_{i}}{\partial s} & \frac{\partial U_{i}}{\partial c_{j}} \\
\frac{\partial V_{1}}{\partial s} & \frac{\partial V_{1}}{\partial c_{j}}
\end{array}\right], \quad i, j=1, \ldots, n-1,
$$

is nonsingular for $|p|$ sufficiently small. This follows from Lemmas 3.5 and 3.6 and the Lemma is proven.

Now we are in a position to construct the extended submanifold. Let $\varepsilon=$ $\min \left(\varepsilon_{0}, \varepsilon_{1}\right)$, where $\varepsilon_{0}, \varepsilon_{1}$ are given by Theorem 3.2 and Lemma 3.7. Let $\widetilde{Q}_{\varepsilon}$ $=Q_{\varepsilon}-S(F)$.

THEOREM 3.8. Let $F$ be the differentiable map defined on $Q_{\varepsilon}$ given by (3.4). Then

(i) $S(F)$ has topological dimension $\leqq n$.

(ii) $F: \widetilde{Q}_{\varepsilon} \rightarrow C^{n}$ is a regular (maximal rank) $C^{k}$ map defining a submanifold immersed in $C^{n}, k \geqq 1$.

(iii) $F \mid P_{\varepsilon} \subset M^{n+1}$.

REMARK. In this theorem we may let $k$ be as large as we please, by choosing $\varepsilon$ sufficiently small.

Proof. Since $J(F)$ is a holomorphic function of $\zeta$ for fixed $(s, c)$, then $J(F)(c, s)$ can only be singular at isolated points in $D$ or singular everywhere in $D$. Since $J(F)$ is not singular at $\zeta=0$ by Lemma 3.7 , then $J(F)$ is nonsingular in $D$ except on a discrete set. Since this is valid for all $(c, s)$, then the set of singularities of $F$ is at most an $n$-dimensional subset of $Q$. The theorem then follows.

This theorem will now give us the main result of this section.

THEOREM 3.9. Let $M \subset C^{n}$ be a $C^{\infty}$ real $(n+1)$-dimensional submanifold of $C^{n}$ with $T C(M)$ nondegenerate and of complex rank 1 at $p \in M$. Suppose, moreover, the Levi form is nonzero at $p$. Then, given any $k \geqq 1$, there exists an $(n+2)$-dimensional real $C^{k}$ submanifold $\tilde{M}$ such that $M$ is extendible to $\tilde{M}$.

Proof. We construct the map

$$
F: Q_{\varepsilon} \rightarrow C^{n}
$$

given by the previous theorem. We have $F(0)=0$. Then $F\left(Q_{\varepsilon}\right)$ is an $n$-parameter continuous family of analytic discs as defined in [18] and by Theorem 1 of that 
same paper we obtain that $M$ is extendible to $F\left(Q_{\varepsilon}\right)$. Let $\tilde{M}$ be $F(N)$ where $N$ is a neighborhood of $q \in \widetilde{Q}_{\varepsilon}$ so that its image in $C^{n}$ is a submanifold embedded in $C^{n}$. The theorem is then proven.

We want to give an example of a 4-dimensional submanifold $M \subset C^{3}$ which satisfies the conditions of Theorem 3.9 but which is not extendible to an open set.

Namely, take

$$
M=\left\{z \in C^{3}:\left|z_{2}\right|=\left|z_{1}\right|-1,\left|z_{2}\right| \leqq \varepsilon,\left|z_{3}\right|=2\right\},
$$

and let $p=(1,0,2)$, for instance. One can check that $L_{p} \neq 0$, and in fact, by standard Cauchy integral techniques it is easy to see that $M$ is extendible to

$$
\tilde{M}=\left\{z \in C^{3}:\left|z_{2}\right| \leqq\left|z_{1}\right|-1,\left|z_{2}\right| \leqq \varepsilon,\left|z_{3}\right|=2\right\}
$$

which is a 5-manifold. But $M^{5}$ can be expressed as the countable intersection of domains of holomorphy $U_{n}$ in $C^{3}$ given by

$$
U_{n}=\left\{z \in C^{3}:\left|z_{2}\right|<\left|z_{1}\right|-\left(1-\varepsilon_{n}\right),\left|z_{2}\right|<\varepsilon+\varepsilon_{n},\left|z_{3}\right|<2+\varepsilon_{n}\right\}
$$

where $\varepsilon_{n}<1$, and $\varepsilon_{n} \rightarrow 0$, as $n \rightarrow \infty$. It follows that $M^{5}$ is not extendible to an open set.

REMARK. Let $M$ be a compact $C^{\infty}$ real $k$-dimensional submanifold of a Stein manifold $X$ with $T C(M)$ of constant complex rank $n>0$. We conjecture that $M$ is extendible, and moreover that $\phi[E(M)]$ contains submanifolds of $X$ of real dimension greater than $k$ ( $\phi$ as in Proposition 1.1). This is true in certain cases. Bishop's peak point theorem [2] implies the existence of points $p \in M$ such that there are no complex submanifolds at any positive dimension embedded in $M$ containing the point $p\left({ }^{4}\right)$. It follows from Theorem 2.3 that, if $k>n, m=k-n$, then $M$ is extendible. Moreover, if $k=n+1$, and $m=1$, it follows from Theorem 3.9 that $M$ is extendible to a manifold of real dimension $n+2$. Also, if $k=2 n-1$, then $M$ is extendible to an open set, which follows from Stein's theorem [16] (see also [9], [18]), on extension from strongly pseudo-convex hypersurfaces. This is, of course, related to Hartog's classical theorem, but the analysis here gives us less information on the extended open set.

Remark (AdDed in Proof). It has been pointed out to me by H. Rossi that the proof of Theorem 3.9 generalizes without much trouble to the case $M^{k} \subset C^{n}, k>n$, and rank $T C\left(M^{k}\right)=k-n$. That is, under these conditions $M^{k}$ is locally extendible to a differentiable submanifold of one higher dimension as in Theorem 3.9. One needs only find the suitable generalization of (3.6) for rank $T C\left(M^{k}\right)>1$, so that the computations can be carried out. The extension manifold will be a $(k-1)$-parameter family of analytic discs, where the parameters come from $w_{2}, \ldots, w_{k-n}$, as given by Iemma 2.5. The estimates of Lemma 3.1 and the corresponding existence theorem (Theorem 3.2) are valid in this case without change. It follows that

(4) This was pointed out to me by H. Rossi. 
Theorem 2.7 and Corollary 2.8 extend to this case. This extension result is also contained in the thesis of S. Greenfield (Brandeis, 1967), where a somewhat different proof is involved (still using the techniques of Bishop, however). Greenfield studies also higher order Levi forms and higher order extendibility of submanifolds.

\section{REFERENCES}

1. E. Bishop, Differentiable manifolds in complex Euclidean space, Duke Math. J. 32 (1965), $1-22$.

2. - A minimal boundary for function algebras, Pacific J. Math. 9 (1959), 629-642.

3. R. Courant and D. Hilbert, Methods of mathematical physics. Vol. II, Interscience, New York, 1962.

4. M. Freeman, "Some conditions for uniform approximation on a manifold" in Function algebras, Scott-Foresman, Chicago, Ill., 1965, pp. 42-65.

5. R. C. Gunning and H. Rossi, Analytic functions of several complex variables, Prentice-Hall, Englewood Cliffs, N. J., 1965.

6. L. Hörmander, An introduction to complex analysis in several variables, Van Nostrand, Princeton, N. J., 1966.

7. H. Kneser, Die Randwerte einer analytischen Funktion zweier Veränderlichen, Monatsh. Math. 43 (1936), 364-380.

8. R. Hermann, Convexity and pseudo convexity for complex manifolds, J. Math. Mech. 13 (1964), 667-672.

9. H. Lewy, On the local character of the solutions of an atypical linear differential equation in three variables and a related theorem for regular functions of two complex variables, Ann. of Math. (2) 64 (1956), 514-522.

10. - On hulls of holomorphy, Comm. Pure Appl. Math. 13 (1960), 589-591.

11. R. Nirenberg and R. Wells, Holomorphic approximation on real submanifolds of a complex manifold, Bull. Amer. Math. Soc. 73 (1967), 378-381.

12. H. Rossi, Holomorphically convex sets in several complex variables, Ann. of Math. (2) 74 (1961), 470-493.

13. - On envelopes of holomorphy, Comm. Pure Appl. Math. 16 (1963), 9-19.

14. J.-P. Serre, Quelques problèmes globaux relatifs aux variétés de Stein, Colloque de Bruxelles, Thone, Liege and Masson, Paris, 1953, pp. 57-68.

15. F. Sommer, Komplex-analytische Blätterung reeler Mannigfaltigkeiten im $C^{n}$, Math. Ann. 136 (1958), 111-133.

16. K. Stein, Zur Theorie der Funktionen mehrerer komplexer Veränderlichen. Die Regularitätshüllen niederdimensionalen Mannigfaltigkeiten, Math. Ann. 114 (1937), 543-569.

17. B. Weinstock, Thesis, Massachusetts Institute of Technology, Cambridge, Mass., 1966.

18. R. Wells, On the local holomorphic hull of a real submanifold in several complex variables, Comm. Pure Appl. Math. 19 (1966), 145-165.

19. — Locally holomorphic sets, J. Analyse Math. 17 (1966), 337-345.

20. - Holomorphic approximation on real-analytic submanifolds of a complex manifold, Proc. Amer. Math. Soc. 17 (1966), 1272-1275.

RICE UNIVERSITY, Houston, TeXas 